\title{
Gallbladder Metastasis from Clear Cell Renal Cell Carcinoma: A Rare Site of Presentation of Metastatic Disease
}

\author{
Ilaria Maggio ${ }^{1}$, Veronica Mollica ${ }^{1}$, Marco Del Governatore ${ }^{2}$, Francesca Giunchi ${ }^{3}$, Maria Massucci ${ }^{1}$, Lisa \\ Manuzzi $^{1}$, Antonio Caira ${ }^{2}$, Michelangelo Fiorentino ${ }^{3}$, Giovanni Brandi ${ }^{4}$, Francesco Massari ${ }^{1}$ \\ ${ }^{1}$ Division of Oncology; ${ }^{2}$ General and Emergency Surgery Unit, Department of Surgical and Medical Sciences; ${ }^{3}$ Department of \\ Pathology; ${ }^{4}$ Oncology Unit, Department of Experimental, Diagnostic and Specialty Medicine; Sant'Orsola-Malpighi Hospital, \\ University of Bologna, 40138 Bologna, Italy.
}

\section{Corresponding Author:}

Dr. Francesco Massari

Email: francesco.massari@aosp.bo.it

This is an Open Access article distributed under the terms of the Creative Commons Attribution License (creativecommons.org/ licenses/by/3.0).

Received : March 14, 2020

Accepted : July 2, 2020

Published : July 20,2020

\begin{abstract}
Background: Gallbladder metastasis from renal cell carcinoma is an extremely rare event, accounting for about less than $1 \%$ of cases. Typically, gallbladder metastasis presents as solitary and metachronous. The clinical presentation can consist in symptoms of biliary colic or acute cholecystitis or it can be discovered as an occasional finding. The management of this unique site of metastasis is usually surgical, with complete removal of the gallbladder. Case Report: We report a case of metastatization to the gallbladder from clear cell renal cell carcinoma about 18 years after removing the primary tumor. The patient underwent radical laparoscopic cholecystectomy. Conclusion: Gallbladder is a rare and peculiar site of metastasis from renal cell carcinoma, it can often be clinically silent and metastatization can occur many years after the end of recommended follow up period for radically resected renal cell carcinoma.
\end{abstract}

Keywords: Cholecystectomy, Colic, Gallbladder Diseases, Kidney Neoplasms, Renal Cell Carcinoma.

\section{Introduction}

Renal cell carcinoma (RCC) is the third most frequent urological cancer, with around 78,000 new diagnoses and 14,700 tumor related deaths in 2019 [1]. Clear cell renal cell carcinoma (ccRCC) is the most common histotype of RCC, accounting for about $75-85 \%$ of cases, while the rest are other histopathological entities classified according to the 2016 World Health Organization classification as non-clear cell RCC [2]. In about $75 \%$ of cases, RCC is diagnosed in a localized stage, while in $25 \%$ of cases it presents metastatic at diagnosis. About $20-50 \%$ of patients with RCC develop metachronous disease [3] and the most common sites of metastasis are lung, liver, adrenal gland, contralateral kidney, bone and brain [4]. An extremely rare site of metastasis is the gallbladder, accounting for about less than $1 \%$ of metastatic sites of RCC [5]. The 5-year overall survival (OS) of patients with ccRCC diagnosed in the early stage is superior to $90 \%$. However, the prognosis for metastatic ccRCC is poor, with a 5-year OS of $12 \%$ [5]. Usually, RCC relapses in the first five years from diagnosis, but some cases of relapse after a long period of time have been described. The management of a solitary metastasis is usually surgical with complete removal of the metastatic lesion. In this case report, we describe the management of solitary metastasis of the gallbladder from ccRCC. Another interesting issue related to this case is long duration of relapse of the disease. 


\section{Case Report}

A 73 years-old Caucasian man came to our attention for the discovery of a metastatic lesion to the gallbladder. His medical history was positive for atrial fibrillation in treatment with anticoagulant therapy, two previous transient ischemic attacks at the age of 50 and 57 years, a transurethral resection of the prostate due to benign prostatic hypertrophy, and a previous traumatic fracture of dorsal vertebrae D8 and D10. In November 2001, the patient underwent right nephrectomy for clear cell renal cell carcinoma, pT1Nx (stage I). After surgery, because of the absence of detectable metastases at computed tomography (CT) scan of chest and abdomen, the patient started attending regular follow-up visits, all negative for disease recurrence for many years. In January 2019, the patient was subjected to an endoscopic retrograde cholangiopancreatography with papillosphincterotomy for cholecystocholedociallithiasis, complicated by duodenal micro-perforation and bleeding, treated with clip positioning and biliary stent. A control CT scan of chest and abdomen with contrast medium performed in April 2019 showed a nodular lesion of $20 \times 27$ millimeters at the fundus of the gallbladder, suggestive for a heteroformative process [Fig.1]. There were no other metastatic lesions. In June 2019, the patient underwent laparoscopic cholecystectomy. The pathological examination revealed infiltration of the gallbladder wall by clear cell carcinoma compatible with renal genesis (immunohistochemistry positive for PAX 8, RCC, CA IX) [Fig.2]. The lesion was entirely contained in the gallbladder wall and a removed pericystic lymphatic node was reactive. Considering that the gallbladder metastasis was solitary without any other site of metastasis at the CT scan, the patient restarted attending regular follow up visits.

\section{Discussion}

In this case report, we describe the metastasis at the gallbladder of ccRCC origin about 18 years from the primary tumor excision. RCC usually metastasizes

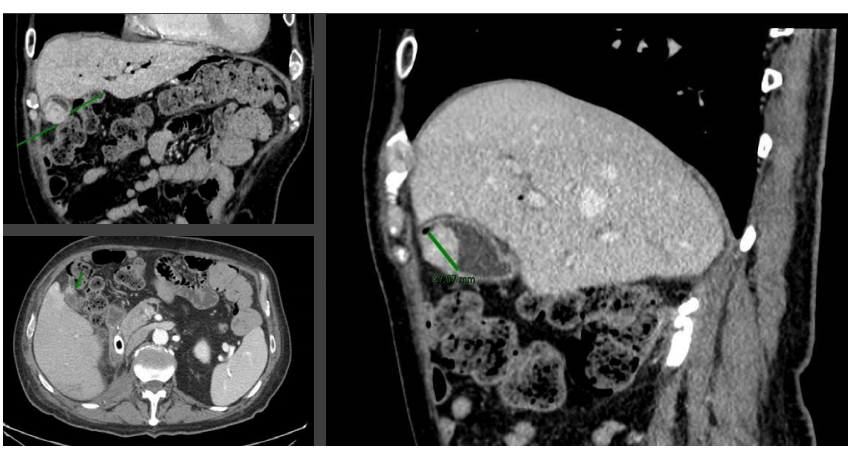

Fig.1: Computed tomography scan images of metastatic lesion to the gallbladder.

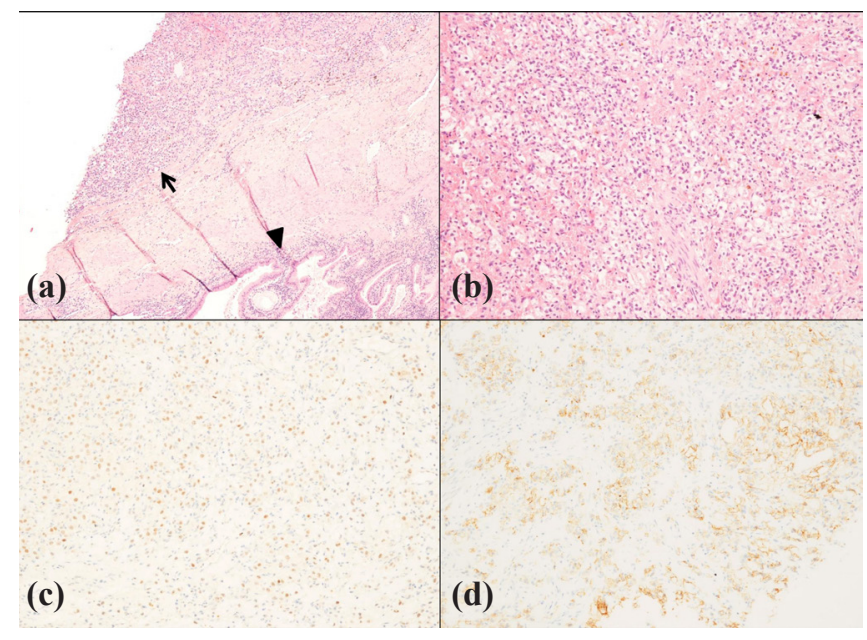

Fig.2 (a): Gallbladder tissue (arrowhead) infiltrated by clear cell renal cell carcinoma (arrow), Hematoxylin \& Eosin $2 X$; (b): Neoplastic cells with clear cytoplasm, Hematoxylin \& Eosin 20X; (c): Immunohistochemistry for PAX8 with nuclear positivity in the neoplastic cell; (d): CAIX cytoplasmatic positivity.

to lung, liver, adrenal gland, contralateral kidney, bone and brain [4]. Metastases at the gallbladder from ccRCC are very uncommon and very few cases have been described in the literature, with a frequency of less than $1 \%$ of cases and even less, around $0.58 \%$, reported in large autopsy reviews $[6,7]$. The detection is often occasional or at post-mortem examination. Rarely, it can present as biliary colic or acute cholecystitis. The silent clinical presentation can be due to the low association of gallstone that has been described in about $28 \%$ of cases [4]. Other primary tumors that can metastasize to the gallbladder are mostly 
melanoma and less frequently lung cancer, pancreas, colon or oesophageal carcinoma [8]. The metastatization process linked to ccRCC seems to be due to direct invasion or a blood spread of tumor cells secondary to vascular invasion [9]. Gallbladder metastasis from RCC occurred mainly in male sex and at a median age of 62 years and the metastasis can originate from either right (57\% of cases) or left kidney ( $43 \%$ of cases). Metastases at the gallbladder have been reported to be solitary in the majority of cases (about 75\%), without any other site of metastasis [4]. The solitary presentation of this peculiar site of metastasis is also characteristic of pancreatic presentation of metastatic disease from RCC that also usually appears to be metachronous [10].

Another interesting issue emerging from this case report is the very long time to relapse. Most guidelines suggest conducting a follow-up after radical excision of $\mathrm{ccRCC}$ for the duration of five years, in consideration that most ccRCC metastases appear metachronously, with a median disease-free interval (DFI) of 4 years [6]. Interestingly, a case of disease relapse at the gallbladder after 27 years has been described [4]. The benefit of long term follow-up has never been demonstrated [11]. When the disease relapses with only a solitary metastasis, the resection of the solitary localized lesion can be curative; in fact, a survival benefit was shown to be associated with surgical removal [12]. Shyr and colleagues showed that single site of first recurrence, curative resection of first metastasis, long DFI, solitary site of first metastasis and metachronous metastases are favorable predictors of survival for metastatic RCC [4]. The study by Neves et al. showed a median DFI of 37 month after cholecystectomy, three and five year OS rates of $74 \%$ and $62 \%$ respectively, and no local site recurrence [6]. Moreover, DFI and single site metastasis are positive prognostic factors [13]. In fact, according to International Metastatic RCC Database Consortium criteria time from diagnosis to treatment is one of the six prognostic factors for metastatic disease associated with OS [14-16].

\section{Conclusion}

Here, we report a case of metastatization to the gallbladder from ccRCC about 18 years after removing the primary tumor. Gallbladder is a rare and unique site of metastasis from ccRCC, it is often clinically silent and it can occur many years after the end of recommended follow up period. A longer DFI from radical treatment of the primary tumor is associated with a better prognosis. If gallbladder metastasis presents as a solitary lesion, surgery can be curative and it is not associated with a poor prognosis advanced disease. Cholecystectomy is the recommended treatment for its outcome and to have a confirmed diagnosis.

Contributors: IM, FM: conceptualization; IM, VM, MDG, FG, MM, LM, AC, MF, GB, FM: data curation; IM, VM, FM writing-original draft preparation; MDG, FG, MM, LM, AC, MF, GB: writing-review and editing. FM will act as a study guarantor. All authors approved the final version of the manuscript and are responsible for all aspects of the study. Funding: None; Competing interests: None stated.

\section{References}

1. Siegel RL, Miller KD, Jemal A. Cancer statistics, 2019. CA Cancer J Clin. 2019;69:7-34.

2. Moch H, Cubilla AL, Humphrey PA, Reuter VE, Ulbright TM. The 2016 WHO classification of tumours of the urinary system and male genital organs-part A: Renal, penile, and testicular tumours. Eur Urol. 2016;70:93105.

3. Sand M, Bechara FG, Kopp J, Krins N, Behringer D, Mann B. Gallbladder metastasis from renal cell carcinoma mimicking acute cholecystitis. Eur J Med Res. 2009;14:90-92.

4. Shyr BU, Chen SC, Shyr YM, Lee RC, Wang SE. Metastatic polyp of the gallbladder from renal cell carcinoma. BMC Cancer. 2017;17:244.

5. Zouari S, Ben Othmen M, Abdessayed N, Larbi Mama N, Jarrar MS, Sriha B, et al. Metastatic renal clear cell carcinoma mimicking a gallbladder polyp: Case report and literature review. Int J Surg Case Rep. 2019;64:133138.

6. Neves MC, Neofytou K, Giakoustidis A, Hazell S, Whoterspoon A, Gore M, et al. Two cases of gallbladder metastasis from renal cell carcinoma and review of literature. World J Surg Oncol. 2016;14:87. 
7. Nojima H, Cho A, Yamamoto H, Nagata M, Takiguchi $\mathrm{N}$, Kainuma $\mathrm{O}$, et al. Renal cell carcinoma with unusual metastasis to the gallbladder. J Hepato-Biliary-Pancreat Surg. 2008;15:209-212.

8. Kawahara T, Ohshiro H, Sekiguchi Z, Furuya M, Namura $\mathrm{K}$, Itoh $\mathrm{H}$, et al. Gallbladder metastasis from renal cell carcinoma. Case Rep Oncol. 2010;3:30-34.

9. Saito Y, Okuda H, Yoshida M, Okimasa S, Fukuda T, Yano M, et al. Gallbladder metastasis of renal clear cell carcinoma 15 years after primary cancer excision: a case report. J Med Case Rep. 2018;12:162.

10. Hung JH, Wang SE, Shyr YM, Su CH, Chen TH, Wu CW. Resection for secondary malignancy of the pancreas. Pancreas. 2012;41:121-129.

11. Dabestani S, Beisland C, Stewart GD, Bensalah K, Gudmundsson E, Lam TB, et al. Long-term outcomes of follow-up for initially localised clear cell renal cell carcinoma: RECUR database analysis. Eur Urol Focus. 2019;5:857-866.

12. White I, Smith S, Rizkalla K, Davies E. Unexpected gallbladder metastasis of clear cell renal carcinoma. Clin Pathol. 2019;12:2632010X19861112.
13. Kavolius JP, Mastorakos DP, Pavlovich C, Russo P, Burt ME, Brady MS. Resection of metastatic renal cell carcinoma. J Clin Oncol. 1998;16:2261-2266.

14. Heng DY, Xie W, Regan MM, Warren MA, Golshayan AR, Sahi C, et al. Prognostic factors for overall survival in patients with metastatic renal cell carcinoma treated with vascular endothelial growth factor-targeted agents: results from a large, multicenter study. J Clin Oncol. 2009;27:5794-5799.

15. Heng DY, Xie W, Regan MM, Harshman LC, Bjarnason GA, Vaishampayan UN, et al. External validation and comparison with other models of the International Metastatic Renal-Cell Carcinoma Database Consortium prognostic model: a population-based study. Lancet Oncol. 2013;14:141-148.

16. Di Nunno V, Mollica V, Schiavina R, Nobili E, Fiorentino $\mathrm{M}$, Brunocilla $\mathrm{E}$, et al. Improving IMDC prognostic prediction through evaluation of initial site of metastasis in patients with metastatic renal cell carcinoma. Clin Genitourin Cancer. 2019;pii:S1558-767330256-3. 